\title{
Bioinformatics approach to predict target genes for dysregulated microRNAs in hepatocellular carcinoma: study on a chemically-induced HCC mouse model
}

\author{
Filippo Del Vecchio ${ }^{1}$, Francesco Gallo², Antinisca Di Marco², Valentina Mastroiaco', Pasquale Caianiello²,
} Francesca Zazzeroni ${ }^{1}$, Edoardo Alesse ${ }^{1}$ and Alessandra Tessitore ${ }^{1 *}$

\begin{abstract}
Background: Hepatocellular carcinoma (HCC) is an aggressive epithelial tumor which shows very poor prognosis and high rate of recurrence, representing an urgent problem for public healthcare. MicroRNAs (miRNAs/miRs) are a class of small, non-coding RNAs that attract great attention because of their role in regulation of processes such as cellular growth, proliferation, apoptosis. Because of the thousands of potential interactions between a single miR and target mRNAs, bioinformatics prediction tools are very useful to facilitate the task for individuating and selecting putative target genes. In this study, we present a chemically-induced HCC mouse model to identify differential expression of miRNAs during the progression of the hepatic injury up to HCC onset. In addition, we describe an established bioinformatics approach to highlight putative target genes and protein interaction networks where they are involved.

Results: We describe four miRs (miR-125a-5p, miR-27a, miR-182, miR-193b) which showed to be differentially expressed in the chemically-induced HCC mouse model. The miRs were subjected to four of the most used predictions tools and 15 predicted target genes were identified. The expression of one (ANK3) among the 15 predicted targets was further validated by immunoblotting. Then, enrichment annotation analysis was performed revealing significant clusters, including some playing a role in ion transporter activity, regulation of receptor protein serine/threonine kinase signaling pathway, protein import into nucleus, regulation of intracellular protein transport, regulation of cell adhesion, growth factor binding, and regulation of TGF-beta/SMAD signaling pathway. A network construction was created and links between the selected miRs, the predicted targets as well as the possible interactions among them and other proteins were built up.
\end{abstract}

Conclusions: In this study, we combined miRNA expression analysis, obtained by an in vivo HCC mouse model, with a bioinformatics-based workflow. New genes, pathways and protein interactions, putatively involved in HCC initiation and progression, were identified and explored.

Keywords: MicroRNA, hepatocellular carcinoma, HCC mouse model, diethylnitrosamine, DEN, target prediction

\footnotetext{
* Correspondence: alessandra.tessitore@univaq.it

${ }^{1}$ Department of Biotechnological and Applied Clinical Sciences, University of

L'Aquila, Via Vetoio, Coppito 2, 67100 L'Aquila, Italy

Full list of author information is available at the end of the article
} 


\section{Background}

Hepatocellular carcinoma (HCC) is a highly aggressive epithelial tumor originating both from mature hepatocytes and stem cells [1]. It is characterized by poor prognosis and very high rate of recurrence. Epidemiological studies indicate that $\mathrm{HCC}$ is the fifth most common cancer and the third most common cause of cancer-related death worldwide [2]. Major risk factors include $\mathrm{HBV} / \mathrm{HCV}$ infection, alcohol/drug abuse, aflatoxin exposure and genetic defects such as primary hemochromatosis and Wilson's disease [3]. The use of animal models helped to better understand the different phases of the entire cancerous process. In this regard, animals' treatment with diethylnitrosamine (DEN) is one of the most frequently used approaches [4]. Diethylnitrosamine is a well-known hepatic carcinogen. At the cellular level, particularly inside the hepatocyte, it acts as an alkylating agent, producing lesions and DNA mutations [5]. A study demonstrated that DEN administration for several weeks induces a rapid cancer development and promotes HCC formation in $100 \%$ of male and $10-30 \%$ of female mice [6]. Literature reports indicate that tumor molecular profile of mice exposed to DEN are comparable to those related to human HCC cases characterized by a poor prognosis [7]. This is the reason why it is considered a typical approach for hepatocarcinogenesis in vivo studies. MicroRNAs (miRNAs) are a class of small, non-coding RNAs that generated a great impact in the molecular biology field. They can negatively regulate the expression of target genes in a post-transcriptional manner, inducing mRNA degradation or inhibiting mRNA translation [8]. After their discovery, miRNAs received enormous attention because of their ability to regulate almost every aspect of cellular functions, such as differentiation, development, apoptosis and proliferation [9]. MiRNA deregulated activity has been described in various pathologies including cancer [10]. In order to make easier the identification of specific target genes, bioinformatics tools have been set-up. They provide the possibility to analyze a particular sequence located at the 5' end of miRNA, called "seed region", in order to predict the most probable genes potentially interacting with it. Although complementarity remains the main feature, the tools take into account other important characteristics such as site accessibility, sequence conservation, multiple binding sites [11]. Bioinformatics tools have greatly improved methods for detection of miRNA targets, due to their ability in quickly processing huge datasets. Looking at literature, some reports describe the exploitation of these algorithms to make prediction about miRNAs-target gene interactions for $\mathrm{HCC}$, but the majority of these studies halted to the miRNA profiling and the validation of target genes for a specific miRNA [12, 13]. In this paper, we moved forward in order to obtain a list of potential genes, all together related to a small group of significantly altered miRNAs in HCC. So, we started to predict putative target genes by making use of relatively different bioinformatics algorithms [14-18]. Secondly, we conducted enrichment annotation analysis to identify functional clusters which could be related to those target genes. Finally, we built up networks to visualize the possible circuits and pathways where the selected miRNAs could be involved, providing a resource for further functional studies on HCC pathogenesis.

\section{Results}

\section{Histological analysis}

Livers from DEN-treated and control mice were subjected to gross anatomical examination and microscopic analysis. Little nodular structures $(0.1-0.2 \mathrm{~cm}$ maximal dimension) were observed in $20 \%$ of mouse livers belonging to 6 months DEN-treated animals (Fig. 1a), whereas all of the mouse livers from 11 months group developed voluminous hepatic nodules (Fig. 1b). In total, fifty two nodular structures with maximal dimensions ranging from 0.3 to $2 \mathrm{~cm}$ were excised. Infiltrating lymphocytes were shown already after 3 months from DEN treatment, indicating the presence of inflammatory processes (Fig. 1d). In particular, $50 \%$ of hepatic tissue samples from 3 months DEN-treated animals showed infiltrating lymphocytes, and this percentage increased up to $70 \%$ in the 6 months DEN-treated group. Regarding the 11 months group, it was observed that $100 \%$ of samples were characterized by lymphocyte infiltration (Fig. 1f). Histological evaluation showed typical dysplastic alterations in samples from 6 months group (Fig. 1e). Moreover, 11 months-hepatic tissues exhibited particular histological features such as hyperaemia, neoangiogenesis, micronodules and wide fibrotic branches, showing a specific feature disrupting the normal hepatic lobular architecture (Fig. 1f).

\section{MiRNAs expression}

Pooled RNAs from whole hepatic tissues of mice sacrificed after 3, 6 and 11 months were subjected to miRNAs' expression analysis. Several miRNAs were found differentially expressed in hepatic tissues from DENtreated animals, with respect to those from control mice, after 3, 6, and 11 months, and in tumors with respect to controls or peritumor liver tissues from DEN-treated animals. Among them, and after a literature review, we focused our analysis on four miRNAs (miR-27a, miR125a-5p, miR-182, miR-193b), whose dysregulated expression was already described in hepatocarcinogenesis [19-26] and also observed in a high-fat diet-induced hepatocarcinogenesis C57BL/6J model we are analyzing (Tessitore et al., "unpublished observations"), further supporting their putative role in liver cancer. We monitored the miRNA expression level during the abovementioned time-points (Table 1). An expression increase 

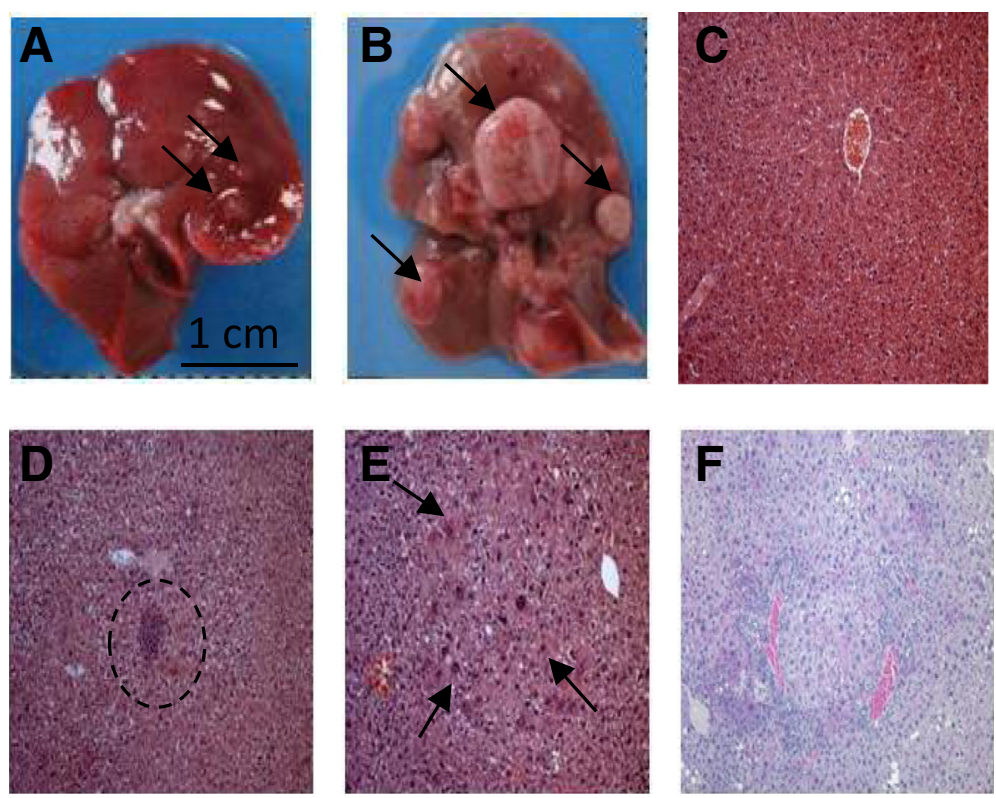

Fig. 1 Progressive liver damage induced by DEN. a-b) Livers from mice sacrificed at 6 (a) and 11 months (b). Dark arrows indicate nodular structures visible on liver's surface. c) H\&E staining showing normal hepatic parenchyma ( $L$ lobe, original magnification 10X) from a control mouse sacrificed after 3 months. d-e) Mouse hepatic tissues (L lobe) from 3- (D, original magnification 10X) and 6-months (E, original magnification 40X) DEN-treated mice. Dashed circle and arrows indicate the presence of infiltrating lymphocytes and cellular atypia. f) Liver parenchyma from an 11-months DEN-treated mouse (L lobe, original magnification 10X). Marked hyperaemia, micronodules and high density of perisinusoidal lymphocytes are detected

of miR-125a-5p, which was up-regulated after 11 months, was detected in DEN mice. MiR-182 was isoexpressed in DEN-treated groups at 3 and 6 months, whereas it was found to be up-regulated at 11 months. MiR-193b appeared iso-expressed or weakly overexpressed during the experimental time points in DENtreated animals. MiR-27a appeared to be down-regulated at 3 months and showed high expression level at 6 and 11 months in DEN-treated mice with respect to controls. MiR-125a-5p, miR-182, miR-193b and miR-27a resulted all over-expressed in tumors in comparison to hepatic tissues from control mice, whereas miRNA125a-5p, miR-182 and miR-193b displayed overexpression or slight increase in the comparison between tumors and paired hepatic tissues from DEN-treated mice.

\section{Target prediction}

A bioinformatics workflow focused on identifying putative target genes with respect to our selected microRNAs was set up and used for the study. A schematic representation is provided in Fig. 2. The first step of analysis involved the construction of a local database (DB) that contained information related to miRNAs and target prediction for Mus musculus (Table 2). The selected microRNAs were analyzed by MiRanda, TargetScan, PITA, and Rna-22, which are some of the most used target prediction tools, in order to identify target genes all at once regulated by the four microRNAs. The first two programs make predictions employing a conservation filter, whereas PITA and Rna-22 are based on freeenergy criteria. MiRanda generated two lists including all targets with a good miRSVR score and a good

Table 1 Relative expression of miRNAs considered for the analysis in livers and tumors

\begin{tabular}{llllll}
\hline & RQ 3 M & RQ 6 M & RQ 11 M & RQ 11 M & RQ 11 M \\
& (DEN/CTR) & (DEN/CTR) & (DEN/CTR) & (DEN-T/CTR) & 1,52 \\
\hline $125 \mathrm{a}-5 \mathrm{p}$ & 0,75 & 0,92 & 2,2 & 3,34 & 2,86 \\
182 & 0,97 & 1,05 & 3,52 & 10,09 & 3,67 \\
$193 \mathrm{~b}$ & 1 & 1,65 & 1,33 & 4,89 & 1,11 \\
$27 \mathrm{a}$ & 0,17 & 15,72 & 4,27 & 4,89 & \\
\hline
\end{tabular}

$\mathrm{RQ}$ value is the relative quantification of miRNA expression, depending on the treatment's length (3, 6, 11 months), obtained by comparing hepatic samples from DEN-treated (DEN), controls (CTR), and tumor samples (DEN-T) from DEN-treated mice, as indicated in the round brackets. Results are mean of 3 iterations 


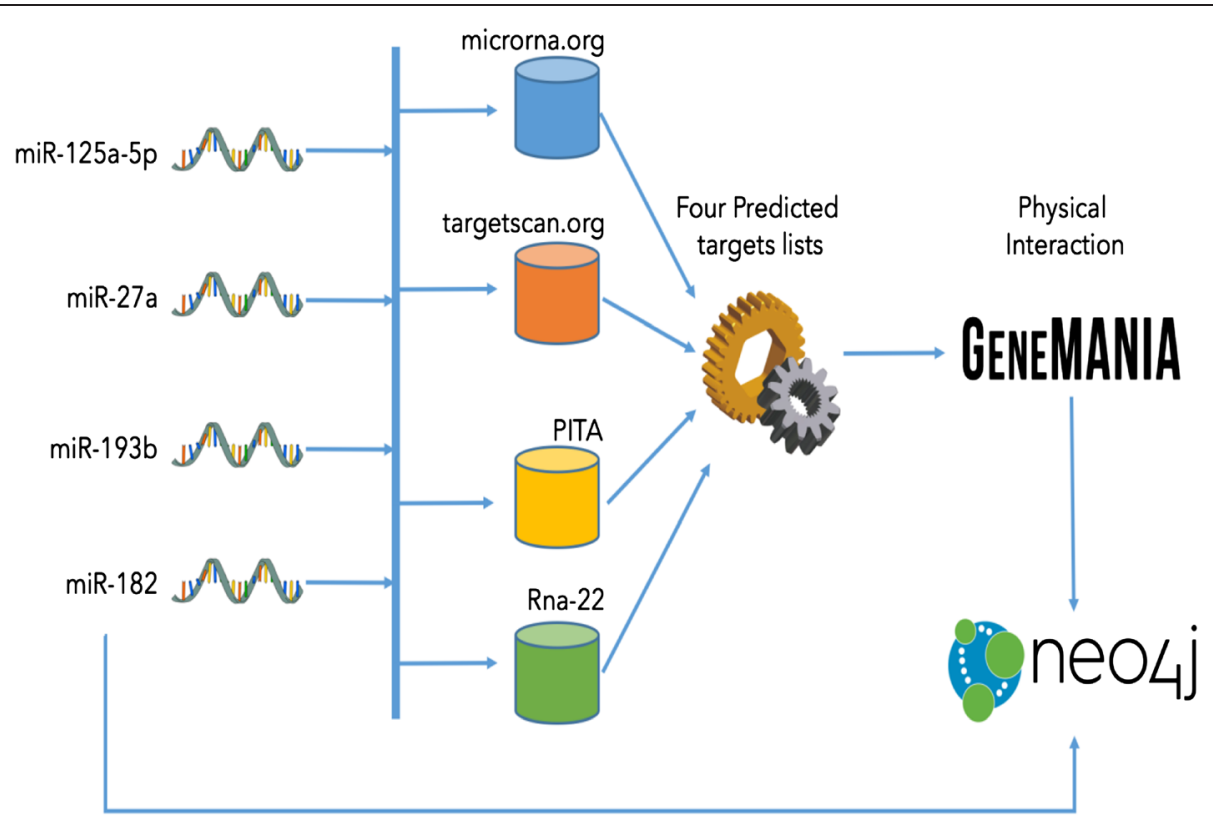

Fig. 2 Workflow of the miRNA analysis. MiR-125a-5p, miR-27a, miR-193b and miR-182 have been considered in our analysis. We look for their common targets on four on-line DB (microrna.org, Targetscan.org, PITA and Rna-22) in order to obtain four predicted targets lists, one for each DB. From these lists, only 15 targets have been considered. We gave the filtered list of targets as input to GENEMANIA in order to obtain a physical relation network. Finally, we built up a network showing the relationships between miRNAs and targets, as well as those among targets by using Neo4j

conservation score, respectively. Then, it searched for links among the lists and created an intersecting final table comprising all target genes with the highest two types of scores. In order to work with stringent criteria and to limit false positive prediction results, a list of 29 "Good miRSVR score, non-conserved miRNA" genes, containing the most probable target genes potentially interacting with those miRNAs, was obtained. Selected microRNAs were then subjected to TargetScan, by taking into consideration the Pct score for measuring site conservation and for better translating the results from mouse to human. From the "Conserved Family Info Result" table, a total number of 148 genes was obtained. PITA and Rna-22 analysis resulted in a final group of 91 and 178 genes, respectively. All the data are described in more detail in an additional file (see Additional file 1: Table S1). For the final analysis, we decided to consider

Table 2 Databases and Tables used for miRNAs/targets interaction analysis

\begin{tabular}{ll}
\hline DB name & Considered table \\
\hline Mirbase Data Base & mirnaTable \\
Microrna Data Base & gcpredSCTable \\
PITA Data Base & PITA_sites_mm9_0_0_ALL table \\
RNA22 Data Base & mus_musculus_ensembl 65 table \\
TargetScan Mouse Data Base & Conserved Family Info table \\
\hline
\end{tabular}

The on-line DB (left) and the respective specific tables considered to extract targets' information (right) are reported only genes predicted by at least 2 of the above-mentioned 4 prediction programs. ANK3 mRNA was the unique target predicted by three different programs (MiRanda, TargetScan, PITA). In addition to ANK3, fourteen mRNAs were predicted by 2 different programs (Fig. 3, Additional file 2: Table S2).

\section{ANKG expression in livers and tumors}

In order to assess the applicability and pertinence of the described workflow, liver tissues from 11 months DENtreated and control mice as well as tumors from 11 months DEN-treated mice were analyzed for the expression of ANKG, which is the protein product of $A N K 3$. ANKG isoforms at 200,170, 120, and $105 \mathrm{kDa}$ have been detected in mouse tissues [27]. Results from immunoblotting (Fig. 4) show a weaker expression of the $170 \mathrm{kDa}$ ANKG isoform in liver tissues from 11 months DEN-treated mice with respect to those from 11 months controls, and a further reduced ANKG expression is observed in tumors from 11 months DEN-treated animals. The results are in line with those evidenced in miRNA expression analysis, which, on the contrary, show a corresponding miRNAs' expression level increase in DEN tissues and tumors. The evidences obtained provide a validation of in silico data.

\section{Enrichment annotation analysis and network construction}

For the network construction and the enrichment annotation step, we took advantage of Genemania [28], which 


\begin{tabular}{|c|c|c|c|c|}
\hline $\begin{array}{l}\text { Target } \\
\text { genes }\end{array}$ & miR-125a-5p & $\overline{\text { miR-27a }}$ & miR-182 & miR-193b \\
\hline \multicolumn{5}{|l|}{ Ank3 } \\
\hline \multicolumn{5}{|l|}{ Tril } \\
\hline \multicolumn{5}{|l|}{ Magi1 } \\
\hline \multicolumn{5}{|l|}{ Acvr2a } \\
\hline \multicolumn{5}{|l|}{ Dtna } \\
\hline \multicolumn{5}{|l|}{ Ikzf3 } \\
\hline \multicolumn{5}{|l|}{ Mll1 } \\
\hline \multicolumn{5}{|l|}{ Mtus1 } \\
\hline \multicolumn{5}{|l|}{$\operatorname{Scn} 2 b$} \\
\hline \multicolumn{5}{|l|}{ Slc8a1 } \\
\hline \multicolumn{5}{|l|}{ Tsc22d2 } \\
\hline \multicolumn{5}{|l|}{ Cyld } \\
\hline \multicolumn{5}{|l|}{ Kcnc1 } \\
\hline \multicolumn{5}{|l|}{ Slc6a17 } \\
\hline Usp24 & & & & \\
\hline
\end{tabular}

Fig. 3 Schematic diagram illustrating the resulting 15 potential top targets for the selected microRNAs. The list includes only genes predicted by at least 2 of 4 prediction tools. Blank boxes represent too low (under the considered cut-off, see "Enrichment annotation analysis and network construction" section in "Materials and Methods") or null association with microRNAs. Genes predicted by miRanda $\square$ genes predicted by TargetScan $\square$ genes predicted by PITA $\square$ genes predicted by Rna-22 $\square$

operated a schematic clusterization of the gene list, also reporting FDR (False Discovery Rate) and gene coverage for each cluster. The results obtained were included in a Neo4J database [29] to provide a graphical representation of miRNAs-targets interactions, including the 15 top target genes as well as 59 secondary genes (Fig. 5). Concerning the 15 top target genes, we extracted 26 significant clusters (FDR $\leq 0.05)$ regarding multiple cellular mechanisms such as ion transporter activity, regulation of receptor protein serine/threonine kinase signaling pathway, protein import into nucleus, regulation of intracellular protein transport, regulation of cell adhesion, growth factor binding, positive regulation of pathway-restricted SMAD protein phosphorylation (see Additional file 3: Table S3). Five genes (SCN2B, SLC8A1, KCNC1, ACVR2A, $C Y L D)$ appear with a "5-times" average in those categories. Moreover, in order to check for significant categories specifically related to hepatic cancer, we extended the analysis to the whole 59 secondary genes set and we obtained 20 significant clusters (Additional file 4: Table S4). In addition, we focused the analysis on a subset of 33 out of 59 secondary genes which, on the basis of PubMed search results, appeared to be specifically described in liver tumor pathogenesis (Additional file 5: Table S5). In this case, we collected 14 significant functional clusters (Additional file 6: Table S6). Regulation of TGF-beta/SMAD signaling pathway, which is known to be involved in liver damage and HCC [30], was evidenced among the most significant and important clusters identified by the above-mentioned data elaborations (Additional file 5: Table S5).

\section{Discussions}

In the present study we show the results of a massive analysis for retrieving possible targets and pathways involved in the initiation and development of chemically-induced hepatocellular carcinoma, by using the C57BL/6J mouse model. The adoption of a combination of microRNAs analysis and computational-based approach allowed us to produce a set of 15 top genes predicted to be the best potential targets for four microRNAs (miR-125a-5p, miR182, miR-193b and miR-27a). The following enrichment annotation analysis, performed both on the 15 top genes and the resulting secondary genes, allowed us to identify, among several pathways and genes involved in, some which plays an important role in liver tumor pathogenesis (i.e., TGF-beta/SMAD signaling pathway). Among the 15 top targets, some protein products have been already described in oncogenesis and metastasis. ANK3 seems to be the most solid candidate, being predicted by 3 out of 4 programs: in particular, it results a suitable target for miR182 , which is greatly up-regulated at 11 months in tumor samples compared to controls. ANK3, in mouse, is distributed in several cell types, especially in renal, hepatic, muscular and nervous tissues, where different isoforms (200, 170,120 , and $105 \mathrm{kDa}$ ) have been detected [27]. Our results show expression decrease of the protein product ankyrin G (170 kDa) in liver tissues from DEN-treated with respect to those from control mice, and a further reduced expression in tumors with respect to DEN peritumor tissues. Data are in line with the contextual expression levels of miRNAs in hepatic tissues and tumors, providing evidence about the effectiveness of the procedure here presented and used. ANK3, member of the ankyrin protein family (ANK1, $A N K 2$ and $A N K 3$ ), is typically known as epithelial ankyrin. Its protein product acts as a bridge between the plasma membrane and cytoskeleton, where it links spectrin-proteins to integral membrane proteins and is involved in regulating cellular functions such as cell motility and proliferation [31]. In several human cancer types, ANK3 appeared to be down-regulated contributing to a poor prognosis [32]. Some authors proposed a possible connection between ANK3 dysregulation and epithelialto-mesenchymal transition (EMT) [33]. They stated that decreased levels of Ankyrin-G in tumoral cells caused by the EMT process could free-up the neurotrophin receptor-interacting melanoma antigen (NRAGE) for translocation into the nucleus, where it could interact with the repressor protein TBX2 to suppress $p 14 A R F$ expression. 


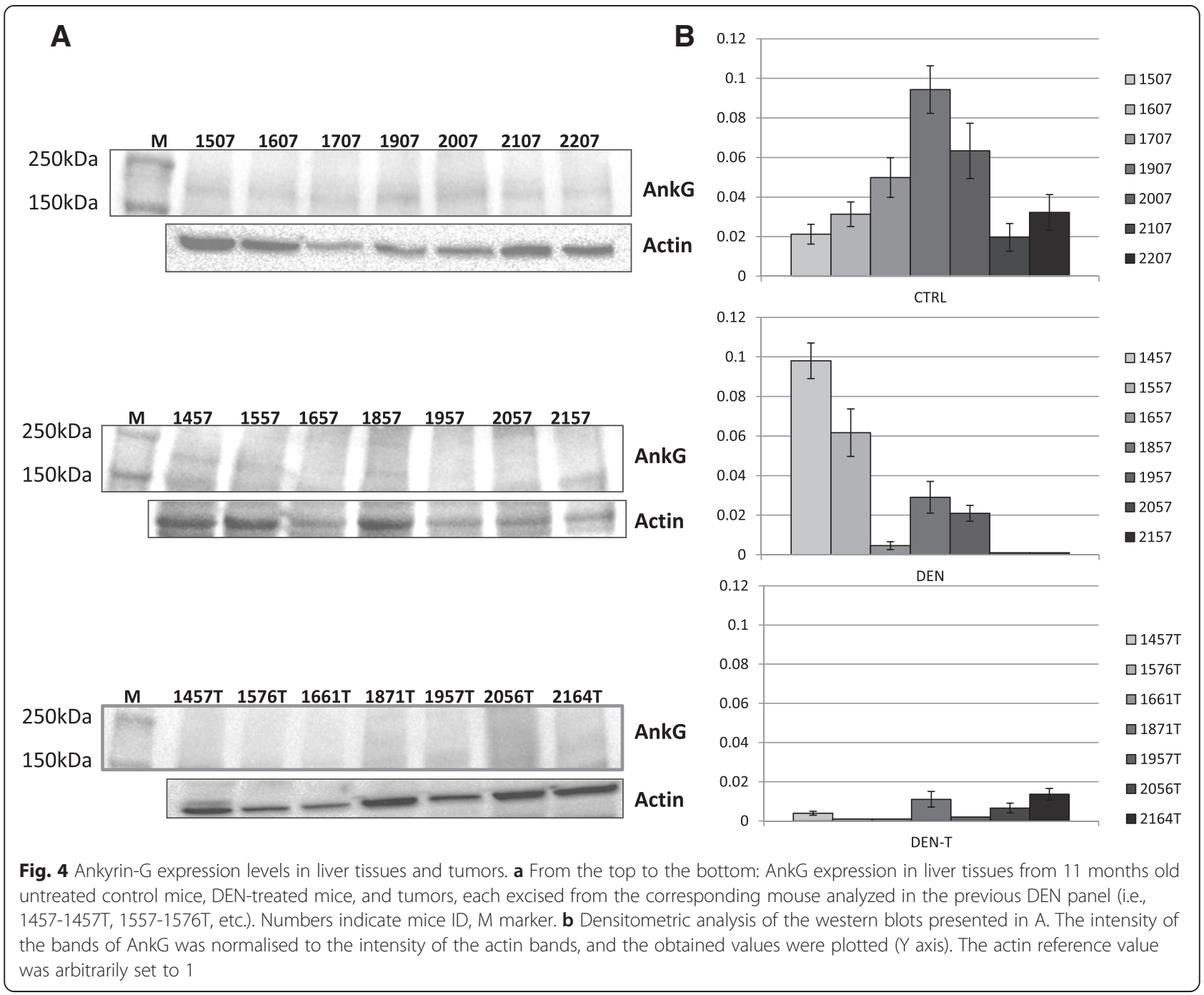

In normal cells, p14ARF acts to block NRAGE-TBX2 complex, allowing NRAGE sequestration by Ankyrin-G. This confers sensitivity to "anoikis", a specific apoptosis program activated when cells are able to detach from extracellular matrix and adhere to other substrates in order to limit their migration potential. Therefore, it could be hypothesized that $P 14 A R F$ down-regulation could protect cancer cells from anoikis activation. An indirect confirmation of the ANK3/EMT relation comes from an interesting work [34] where the role of HOOK1, an interaction ANK3 partner we previously observed in Genemania network (see "Enrichment annotation analysis and network construction" section in "Materials and Methods"), was described in this phenomenon. The authors showed that HOOK1 displayed a regulatory effect on EMT, since its overexpression led to EMT inhibition. Conversely, reduced HOOK1 expression level contributed to EMT phenomenon. The existence of a physical link for Ankyrin G and HOOK1 provide thus an additional clue to the hypothesized role of ANK3 in the regulation of EMT for tumor metastases [35]. Another compelling mechanism involving a possible role of $A N K 3$ in cancer pathogenesis is presented by an elegant work by Ignatiuk et al. [36] where it was demonstrated that smaller isoforms of ANK3, called ANK120 and ANK105, were able to bind the PDGFR-binding subunit of PI3K, p85, influencing the lysosomal degradation of the receptor. These proteins, particularly expressed in liver, lack the repeat domain which is responsible for their positioning on plasma membrane and for this reason they localize to late endosomes and lysosomes to target materials to be degraded [37]. ANK120 and-105 overexpressing-cells exhibited a rapid degradation followed by reduced levels of PDGF receptor and an overall minor sensitivity to proliferation upon PDGF stimulation. In HCC cells, down-regulation of these isoforms could lead to delayed PDGFR degradation that could give rise to a sustained signalling of PDGF and downstream pathways, resulting in enhanced proliferation. Keeping in mind that PDGFR expression is reported to be up- 


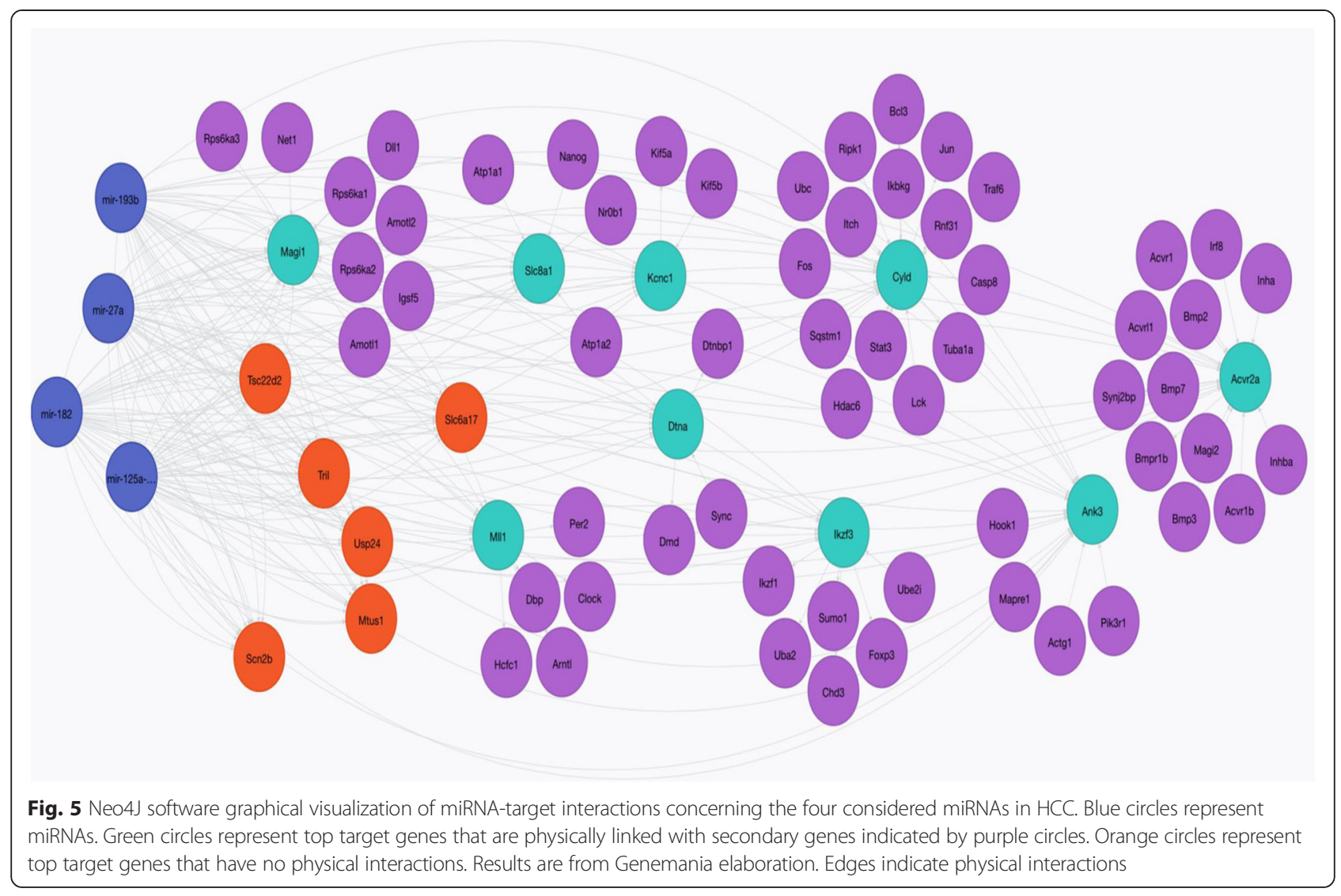

regulated in HCC human livers [38], focused analysis of this mechanism could be very intriguing to explore.

CYLD, here identified as a potential miRNAs target and in functional clusterization, is a protein implicated in the regulation of protein localization and transport inside the cells. CYLD is a gene whose loss or mutations predispose to the development of human cylindroma, a particular type of hair follicle benign tumor. It encodes for a deubiquitinating enzyme, ubiquitously expressed, which is depicted as a tumor suppressor since it lacks its expression in various human tumor types [39]. CYLD removes ubiquitin chains from different molecules, such as TNF receptor-associated factor $2 / 6$ (TRAF2/6) and Bcell limphoma-3 (BCL-3), thus participating in pathways involved in cell proliferation and survival [40]. Literature reports illustrated some mechanisms specifically involving this gene in tumor tissues. Pannem et al. [41] showed that CYLD-deficent mice developed hepatic tumors after DEN-treatment, using an animal model similar to that here described. At the molecular level, CYLD downregulation led to robust activation of JNK1 which reflected in $c-M y c$ levels stabilization and enhanced transcription of cyclin-D1. This mechanism could justify the elevated proliferation rates observed in HCC cells. On the other hand, other researchers demonstrated also direct CYLD crosstalk with NF-kB pathway [42]. In this case, CYLD influenced this pathway through the control of BCL-3 localization. Indeed, BCL-3 was able to associate with NF$\mathrm{kB}$ heterodimers and triggered the transcription of NF$\mathrm{kB}$-related genes such as cyclin-D1, but only when it was inside the nucleus [43]. In normal cells, BCL-3 was retained into the cytoplasm, due to the elimination of Lys63 polyubiquitin chains, a task mediated by CYLD itself. During transformation, growth-promoting stimuli produced BCL-3 transfer from the cytoplasm to the perinuclear region. Here, decreasing $C Y L D$ levels activated BCL3 accumulation followed by its import into the nucleus, probably mediated by an interaction between the polyubiquitin chains of $\mathrm{BCL}-3$ and importins. Once inside the nucleus, BCL-3 can bind to NF-kB to form a complex that was able to selectively recognize NF-kB binding sites, stimulating trascription of oncogenes. Based on our findings, $C Y L D$ was predicted to be a target of miR-125a-5p and miR-182. Effectively, CYLD is validated for human miR-182, according to miRTarBase (ISBLab) [44]. In light of all these evidences, we can deduce that miR-125a-5p and miR-182 could modify CYLD expression in cancerous tissues causing a marked reduction.

Clusterization analysis reveals the presence of SLC8A1 as an additional gene targeted by the selected microRNAs. Our data showed that this gene could be regulated by miR-182 and miR-27a which have been already 
described as over-expressed microRNAs in human HCC cells $[20,24,25]$. SLC8A1 belongs to the large family of solute carrier (SLC) transporters, proteins designated to transport different molecules including nutrients, metals, ions and drugs across the membranes [45]. Generally, they are influx transporters and SLC $8 A 1$ represents one of them. This gene encodes a protein, called NCX1, which regulates the extra- and intracellular levels of sodium $\left(\mathrm{Na}^{+}\right)$and calcium $\left(\mathrm{Ca}^{++}\right)$, allowing $\mathrm{Na}^{+}$influx and $\mathrm{Ca}^{++}$efflux in normal conditions [46]. NCX1, known as an important $\mathrm{Ca}^{++}$exchanger in plasma membrane, could invert this flux in pathological conditions, determining $\mathrm{Ca}^{++}$influx. This situation provokes a rise of intracellular calcium levels and the consequent activation of $\mathrm{Ca}^{++}$-dependent signaling pathways that stimulate specific responses, such as apoptosis activation. In some human cancers, SLC8A1 expression was shown to be decreased, producing a corresponding reduction of intracellular calcium levels which leads to apoptosis evasion and more sustained proliferation rate [47]. Moreover, as described by Januchowski et al. [48], SLC8A1 was addressed as responsible for multidrug resistance, one of the big problems in chemotherapeutic treatments. In this context, the authors documented a strong SLC8A1 downregulation in several drug-resistant ovarian cancer cell lines, claiming that it could represent an interesting therapeutic target for this malignancy. These data fit with our results; indeed, miR-27a and miR-182 up-regulation might be responsible of SLC8A1 ipo-expression in HCC tissues. Although there are no evidences in literature about SLC $8 A 1$ expression level in HCC, a number of studies have described its lowered activity in other human cancers [47, 48]. Hence, it could be useful to evaluate its expression also in another completely different environment, like HCC could be.

Another interesting gene linked to our group of selected miRNAs and comprised in the 15 top target gene set is MAGI1 (membrane-associated guanylate kinase, WW and PDZ domain containing 1), which is a scaffold protein whose principal activity is to regulate and stabilize cell-cell contacts [49]. Because of this activity, MAGI1 is considered an important regulator for cell junctions with tumor suppressor behaviour. Some studies elucidated its functions stating that it recruits PTEN to the E-cadherin complexes located at junctional sites and, subsequently, induce PTEN to downregulate phosphatidylinositol-3,4,5 trisphosphate pools, leading to the activation of the effector downstream molecules [50]. Zhang et al. reported MAGI1 downregulation at mRNA and protein level in HCC human tissues and they found a direct correlation between its reduced expression and a poor prognosis [51]. These results fitted with our predictions. In fact, our data indicated MAGI1 as a putative target for all of the four miRNAs, in particular for miR-182. Considering that all of these miRNAs were up-regulated in tumor tissues in our system, we could speculate that one or more of them could block the expression of MAGI1 in HCC tissues.

\section{Conclusions}

Since their discovery, many advances were made about the understanding of microRNA functions and their possible use for biomedical purposes. Especially for cancer, an increasing number of studies highlighted their potential in defining diagnosis, prognosis and therapy and great efforts are being made to support the use of these molecules in clinical applications. In this work we combined biomolecular results, based on an in vivo model, with in silico analysis. We created a workflow able to connect miRNAs, found to be dysregulated in chemicallyinduced hepatocarcinogenesis, to respective putative targets; then we generated a wide protein interaction network involving other proteins physically interacting with them. The workflow was validated by evaluating and confirming the expression levels of one among the microRNAs' predicted targets (ANKG) in the animal model here described. In conclusion, the experimental procedure could be used and employed for further researches on HCC initiation, development and progression.

\section{Methods}

\section{Animal husbandry}

Three groups, each composed by eight 14 days old C57BL/6J mice (Charles River laboratories, France) were treated with intra-peritoneal injection of diethylnitrosamine DEN $(25 \mathrm{mg} / \mathrm{kg})$ in saline solution, and sacrificed by $\mathrm{CO}_{2}$ asphyxiation after 3, 6, and 11 months. As many groups of as many 14 days old mice were treated, in parallel, with intra-peritoneal physiological saline solution injection, and sacrificed at the same experimental time points. Animals were fed with a standard diet (Harlan 2918, Teklad Global $18 \%$ Protein Rodent Diet) and housed under the same conditions $\left(\mathrm{T}=20-21{ }^{\circ} \mathrm{C}, 12 \mathrm{~h}\right.$ light-dark cycle). All experimental procedures were performed in conformity with national and international laws and policies (European Economic Community Council Directive 86/609, Italian Legislative Decree 116/ 92, National Institutes of Health Guide for the Care and Use of Laboratory Animals) and were approved by the Internal Committee and the Italian Ministry of Health.

\section{Tissue collection}

After sacrifice, livers were explanted and weighed. Liver tissues were stored in RNA Later (Ambion), frozen at $-80{ }^{\circ} \mathrm{C}$ or fixed in formalin for further analyses. 


\section{Histological analysis}

Immediately after removal, hepatic tissue sections were fixed in $10 \%$ neutral buffered formalin. (formaldehyde $4 \%$ w/m, phosphate buffer 0,05M, Bio Optica). After 48 h, tissue sections were paraffin-embedded using standard procedures. Finally, $3 \mu \mathrm{m}$ sections were stained with hematoxylin and eosin and then observed by light microscope (Nikon Eclipse E200) to visualize the general hepatic architecture.

\section{RNA extraction}

Total RNA was extracted by using MiRVana kit (Ambion) which allows the recovery of total RNA, including the fraction less than $200 \mathrm{nt}$ in lenght, according to the manufacturer's instructions. RNA quantification and purity was measured with Nanodrop 1000 v3.7 (Thermo Scientific) and RNA quality was verified by ethidium bromide staining, after agarose gel electrophoresis.

\section{MiRNA analysis}

Same amounts of total RNA, extracted by livers from eight mice belonging to every experimental group, were mixed together to obtain pools from DEN-treated and control mice. RNAs were also extracted from 7 tumors from 11 months DEN-treated mice (1 tumor per mouse, tumors from the eighth mouse were too small to be excised -2 mm maximal dimension-), and pooled together as well. For the analysis, 700 ng of mixed RNAs were subjected to retro-transcription by using the TaqMan MicroRNA RT kit and the Megaplex Rodent Primer Pool A (Life Technologies), and analyzed for microRNAs' expression levels by using the TaqMan rodent MicroRNA array set A, v3.0 (Life Technologies), according to the manufacturer's instructions. Mamm-U6 was used as endogenous control. Each RNAs' pool was analyzed in triplicate. To collect data, ViiA-7 system and software (Life Technologies) was used, and the $\Delta \Delta \mathrm{Ct}$ comparative method was applied. We considered Relative Quantification values $\mathrm{RQ} \geq 2$ or $\mathrm{RQ} \leq 0.5$ as up- and down-regulation cut-off, respectively.

\section{Western blot}

Tissues were homogenized by using Tissue Lyser (Qiagen), and proteins were extracted in RIPA buffer. Proteins from livers and tumor tissues were recovered from the same samples used for miRNA analysis. We analyzed, at the individual level, seven samples from 11 months controls, DEN-treated mice, and tumors from paired DEN-treated mice. Thirty micrograms of total extracts were loaded onto a 4-15\% SDS-PAGE precast get (Bio-Rad). After electroblotting ( $250 \mathrm{~mA}, 1.5 \mathrm{~h})$, proteins were stained with Ponceau, and membrane was washed, blocked with $5 \%$ non-fat milk in TBST $1 \mathrm{X}$ for $1 \mathrm{~h}$, and then incubated overnight at $4{ }^{\circ} \mathrm{C}$ with 1:500 anti-ANKG antibody (Santa-Cruz) in TBST 1X. The membrane was washed and incubated with the secondary anti-rabbit antibody (Santa Cruz) for $1.5 \mathrm{~h}$ at room temperature. After washing, SuperSignal West Pico Chemiluminescent Substrate (Thermo Scientific) was added and images were collected by using CGel Doc XR+ (Bio Rad) instrument, by using the same exposure time. Densitometric analysis was performed by using Image Lab 4.0 (Bio-Rad). Actin (Santa Cruz) was used as endogenous control, by following the same experimental conditions.

\section{Target prediction analysis}

Four miRNAs (miR-125a-5p, miR-182, miR-193b and miR27a) were selected and subjected to target prediction. MySQL technology [52] was used to construct a local database. In particular, Mirbase [53] has been considered to extract data about microRNA, whereas four on-line DB were used to perform predictive analysis: MiRanda (August 2010 release) [14], TargetScan Mouse v6.2 (June 2012 release) [16-54], PITA (Segal lab of computational biology) [17], and Rna-22 (Thomas Jefferson University) [18].

According to the MiRanda authors' discussion in [15], 1.2 was chosen as cut-off mirSVR score to obtain a reasonable empirical probability of target mRNA downregulation. Data were downloaded from the MiRanda database MirBase. For TargetScan, only genes with a probability of conserved targeting $(\mathrm{Pct})>0.1$ were considered. Data from PITA were recovered downloading the " $3 / 15$ flank All" table in "Mouse" column in "PITA Targets catalog" and setting $10 \mathrm{kcal} / \mathrm{mol}$ as a general cutoff value. Regarding Rna22 , only genes with a binding energy less than $-25 \mathrm{kcal} / \mathrm{mol}$ were included in the subsequent steps.

\section{Network construction and enrichment annotation analysis}

A list constituted by primary target genes was subjected to software analysis as a query list using the software Genemania v3.1.2 and then an interaction network was retrieved, selecting molecular functions as weighing method (dysplaying n.100 related genes and n.20 related attributes in the "number of gene results" field). Similar lists were built by considering also the set of 59 secondary genes and a subset of 33 genes which, on the basis of a PubMed search, appeared already described in liver cancer. Due to the higher number of input genes, the Number Gene parameters were, in this case, reduced to 10 related attributes and 10 related genes. Gene targets network construction was made using the same software Genemania v3.1.2. In particular, physical interactions between targets were considered. The Genemania "Functions" panel that allows the user to visualize all the clusters for Gene Ontology (GO) terms related to the query gene list was used. Thus, this possibility was exploited to carry out enrichment annotation analyses for our 
target genes list. The results hitherto obtained were included in a Neo4J database to provide a graphical representation of miRNAs-targets interactions (Fig. 5).

\section{Additional files}

Additional file 1: Table S1. List of target genes obtained from

TargetScan Mouse v 6.2, List of potential targets from PITA, List of target from Rna-22, List of target genes predicted by MiRanda. Four Excel sheets showing the four lists of potential targets for the miR 125a-5p, miR-193b, miR-182 and miR-27a obtained from the prediction softwares TargetScan Mouse, PITA, Rna-22 and MiRanda. (XLSX $32 \mathrm{~kb}$ )

Additional file 2: Table S2. The group of fifteen top target genes inferred from target prediction analysis. A table comprising the fifteen top target genes for the miR 125a-5p, miR-193b, miR-182 and miR-27a, retrieved from at least $50 \%$ of prediction softwares used. (DOCX $11 \mathrm{~kb}$ )

Additional file 3: Table S3. Most significant functional groups obtained from clusterization process by Genemania ( $F D R \leq 0.05$ ). Excel table presenting the 26 most significant cluster extracted by Genemania after selection of the fifteen top target genes. The table shows the false discovery rate and the gene coverage for each cluster. (XLSX $14 \mathrm{~kb}$ )

Additional file 4: Table S4. Most significant functional groups obtained from clusterization process by Genemania, regarding 59 secondary genes (FDR $\leq 0.05$ ). Excel table presenting the 20 most significant cluster extracted by Genemania on the whole set of 59 secondary genes. The table shows the false discovery rate and the gene coverage for each cluster. (XLSX $11 \mathrm{~kb}$ )

Additional file 5: Table S5. List of secondary genes from Genemania involved in liver cancer on the basis of literature (PubMed) review. Excel table presenting 33 out of 59 secondary genes, already described in liver tumor pathogenesis. (XLSX $9 \mathrm{~kb}$ )

Additional file 6: Table S6. Most significant functional groups obtained from clusterization process by Genemania, regarding 33 secondary genes, most closely related to liver cancer ( $F D R \leq 0.05$ ). Excel table presenting the 14 most significant cluster extracted by Genemania after selection of the 33 secondary genes. The table shows the false discovery rate and the gene coverage for each cluster. (XLSX $11 \mathrm{~kb})$

\section{Abbreviations}

HCC: hepatocellular carcinoma; miRNAs/miRs: microRNAs;

DEN: diethylnitrosamine; DB: database; FDR: false discovery rate; EMT: epithelial-to-mesenchymal transition; NRAGE: neurotrophin receptorinteracting melanoma antigen; BCL-3: B-cell limphoma-3; Pct: probability of conserved targeting; GO: gene ontology.

\section{Competing interests}

The authors declare that they have no competing interests.

\section{Authors' contributions}

FDV is the lead writer and conducted histological analyses, western blot, performed enrichment annotation analysis and assisted in network construction. FG was involved in the construction of the database containing the target genes and the four miRNAs considered, in the extraction and filtration of data from the online database and in the graph representation. ADM contributed to the specification of the bioinformatics project and relative workflow, to the manuscript writing and its revision. VM handled mice, collected tissues and miRNA qRT-PCR data. PC contributed to the specification of the bioinformatics projects and to the manuscript revision. FZ set-up the DEN mouse model and revised the manuscript. EA conceived the project and revised the manuscript. AT conceived the project, processed and analyzed miRNA qRT-PCR data, revised the manuscript. All authors read and approved the final manuscript.

\section{Acknowledgements}

This work was supported by MIUR-FIRB grant n. RBAP10A9H9 to Edoardo Alesse. This work has been done in the context of ARES (mirnAs' influence on canceR dESease), a Class C Project (code: HP10CV8XPV) funded by CINECA ISCRA.

\section{Author details}

'Department of Biotechnological and Applied Clinical Sciences, University of L'Aquila, Via Vetoio, Coppito 2, 67100 L'Aquila, Italy. ${ }^{2}$ Department of Computer Engineering and Science, and Mathematics, University of L'Aquila, Via Vetoio, Coppito 1, L'Aquila 67100, Italy.

Received: 6 August 2015 Accepted: 26 November 2015

Published online: 10 December 2015

\section{References}

1. Vann Malestein H, Van Pelt J, Verslype C. Molecular classification of hepatocellular carcinoma 2011. Eur J Cancer. 2011;47:1789-97.

2. Bosetti C, Turati F, La Vecchia C. Hepatocellular carcinoma epidemiology. Best Pract Res Clin Gastroenterol. 2014;28(5):753-70.

3. Hill-Baskin AE, Markiewski MM, Buchner DA, Shao H, De Santis D, Hsiao G, et al. Diet-induced hepatocellular carcinoma in genetically predisposed mice. Hum Mol Gen. 2009:18(16):2975-88.

4. Fausto N, Campbell JS. Mouse models of hepatocellular carcinoma. Semin Liver Dis. 2010;30(1):87-98.

5. Verna L, Whysner J, Williams GM. N-nitrosodiethylamine mechanistic data and risk assessment: bioactivation, DNA-adduct formation, mutagenicity, and tumor initiation. PharmacolTher. 1996;71(1-2):57-81.

6. Nakatani T, Roy G, Fujimoto N, Asahara T, Ito A. Sex hormone dependency of diethylnitrosamine-induced liver tumors in mice and chemoprevention by leuprorelin. Jpn J Cancer Res. 2001;92(3):249-56.

7. Lee JS, Chu IS, Mikaelyan A, Calvisi DF, Heo J, Reddy JK, et al. Application of comparative functional genomics to identify best-fit mouse models to study human cancer. Nat Genet. 2004;36(12):1306-11.

8. Carrington JC, Ambros V. Role of microRNAs in plant and animal development. Science. 2003:301(5631):336-8

9. Ambros V. The functions of animal microRNAs. Nature. 2004;431 (7006):350-5.

10. Gong H, Liu CM, Liu DP, Liang CC. The role of small RNAs in human diseases: potential troublemaker and therapeutic tool. Med Res Rev. 2005; 25(3):361-81.

11. Saito T, Saetrom P. MicroRNAs - targeting and target prediction. N Biotecnol. 2010;27(3):243-9.

12. Bae HJ, Jung KH, Eun JW, Shen Q, Kim HS, Park SJ, et al. MicroRNA-221 governs tumor suppressor HDAC6 to potentiate malignant progression of liver cancer. J Hepatol. 2015. doi:10.1016/j.jhep.2015.03.019.

13. Chang RM, Yang H, Fang F, Xu JF, Yang LY. MicroRNA-331-3p promotes proliferation and metastasis of hepatocellular carcinoma by targeting $\mathrm{PH}$ domain and leucine-rich repeat protein phosphatase. Hepatology. 2014; 60(4):1251-63

14. Betel D, Wilson M, Gabow A, Marks DS, Sander C. The microRNA.org resource: targets and expression. Nucleic Acids Res. 2008;36:D149-153.

15. Betel D, Koppal A, Agius P, Sander C, Leslie C. Comprehensive modeling of microRNA targets predicts functional non-conserved and non-canonical sites. Genome Biol. 2010;11(8):R90.

16. Friedman RC, Farh KK, Burge CB, Bartel DP. Most mammalian mRNAs are conserved targets of microRNAs. Genome Res. 2009;19(1):92-105.

17. Kertesz M, lovino N, Unnerstall U, Gaul U, Segal E. The role of site accessibility in microRNA target recognition. Nat Genet. 2007:39(10):1278-84

18. Miranda KC, Huynh T, Tay Y, Ang YS, Tam WL, Thomson AM. et al.:A patternbased method for the identification of MicroRNA binding sites and their corresponding heteroduplexes. Cell. 2006;126(6):1203-17.

19. Wu XJ, Li Y, Liu D, Zhao LD, Bai B, Xue MH. MiR-27a as an oncogenic microRNA of hepatitis B virus- related hepatocellular carcinoma. Asian Pac J Cancer Prev. 2013;14(2):885-9. 2013.

20. Huang $S$, He X, Ding J, Liang L, Zhao Y, Zhang Z, et al. Upregulation of miR23a $\sim 27$ a $\sim 24$ decreases transforming growth factor-beta-induced tumorsuppressive activities in human hepatocellular carcinoma cells. Int J Cancer. 2008;123(4):972-8.

21. Braconi C, Valeri N, Gasparini P, Huang N, Taccioli C, Nuovo G, et al. Hepatitis C virus proteins modulate microRNA expression and chemosensitivity in malignant hepatocytes. Clin Cancer Res. 2010;16(3):957-66.

22. Kim JK, Noh JH, Jung KH, Eun JW, Bae HJ, Kim MG, et al. Sirtuin7 oncogenic potential in human hepatocellular carcinoma and its regulation by the tumor suppressors MiR-125a-5p and MiR-125b. Hepatology. 2013;57(3):1055-67. 
23. Wang J, Li J, Shen J, Wang C, Yang L, Zhang X. MicroRNA-182 downregulates metastasis suppressor 1 and contributes to metastasis of hepatocellular carcinoma. BMC Cancer. 2012;12:227.

24. Wang C, Ren R, Hu H, Tan C, Han M, Wang X. MiR-182 is up-regulated and targeting Cebpa in hepatocellular carcinoma. Chin J Cancer Res. 2014;26(1):17-29.

25. Wang TH, Yeh CT, Ho JY, Ng KF, Chen TC. OncomiR miR-96 and miR-182 promote cell proliferation and invasion through targeting ephrinA5 in hepatocellular carcinoma. Mol Carcinog. 2015. doi:10.1002/mc.22286.

26. Leung WK, He M, Chan AW, Law PT, Wong N. Wnt/B-Catenin activates MiR183/96/182 expression in hepatocellular carcinoma that promotes cell invasion. Cancer Lett. 2015;362(1):97-105.

27. Peters LL, John KM, Lu FM, Eicher EM, Higgins A, Yialamas M. Ank3 (Epithelial Ankyrin), a Widely Distributed New Member of the Ankyrin Gene Family and the Major Ankyrin in Kidney, Is Expressed in Alternatively Spliced Forms, Including Forms That Lack the Repeat Domain. J Cell Biol. 1995; 130(2):313-30.

28. Montojo J, Zuberi K, Rodriguez H, Kazi F, Wright G, Donaldson SL, et al. GeneMANIA Cytoscape plugin: fast gene function predictions on the desktop. Bioinformatics. 2010;26(22):2927-8.

29. Robinson I, Webber J, Eifrem E. Graph Databases. 2nd ed. Sebastopol, CA USA: O'Reilly Media, Inc., 2013.

30. Dooley S, ten Dijke P. TGF- $\beta$ in progression of liver disease. Cell Tissue Res. 2012;347(1):245-56.

31. Lambert $\mathrm{S}$, Bennett $\mathrm{V}$. From anemia to cerebellar dysfunction. A review of the ankyrin gene family. Eur J Biochem. 1993;211(1-2):1-6.

32. Glinski GV, Berezovska O, Glinskii AB. Microarray analysis identifies a deathfrom cancer signature predicting therapy failure in patients with multiple types of cancer. J Clin Invest. 2005;115:1503-21.

33. Kumar S, Park SH, Cieply B, Schupp J, Killiam E, Zhang F, et al. A Pathway for the Control of Anoikis Sensitivity by E-Cadherin and Epithelial-toMesenchymal Transition. Mol Cell Biol. 2011;31(19):4036-51.

34. Li S, Wang L, Zhao Q, Liu Y, He L, Xu Q, et al. SHP2 positively regulates TGF 1 -induced epithelial-mesenchymal transition modulated by its novel interacting protein Hook1. J Biol Chem. 2014;289(49):34152-60.

35. Weimer JM, Chattopadhyay S, Custer AW, Pearce DA. Elevation of Hook1 in a disease model of Batten disease does not affect a novel interaction between Ankyrin G and Hook1. Biochem Biophys Res Commun. 2005;330(4):1176-81.

36. Ignatiuk A, Quickfall JP, Hawrysh AD, Chamberlain MD, Anderson DH. The smaller isoforms of ankyrin 3 bind to the p85 subunit of phosphatidylinositol 3' kinase and enhance platelet-derived growth factor receptor down-regulation. J Biol Chem. 2006;281(9):5956-64.

37. Hoock TC, Peters LL, Lux SE. Isoforms of ankyrin-3 that lack the NH2terminal repeats associate with mouse macrophage lysosomes. J Cell Biol. 1997;136(5):1059-70.

38. Chu JS, Ge FJ, Zhang B, Wang Y, Silvestris N, Liu L, et al. Expression and prognostic value of VEGFR-2, PDGFR- $\beta$, and c-Met in advanced hepatocellular carcinoma. J Exp Clin Cancer Res. 2013;32:16.

39. Kinoshita H, Okabe H, Beppu T, Chikamoto A, Hayashi H, Imai K, et al. CYLD downregulation is correlated with tumor development in patients with hepatocellular Carcinoma. Mol Clin Oncol. 2013;1(2):309-14.

40. Massoumi R. Ubiquitin chain cleavage: CYLD at work. Trends Biochem Sci. 2010;35(7):392-9.

41. Pannem RR, Dorn C, Ahlqvist K, Bosserhoff AK, Hellerbrand C, Massoumi R. CYLD controls C-MYC expression through the JNK-dependent signaling pathway in hepatocellular carcinoma. Carcinogenesis. 2014;35(2):461-8.

42. Massoumi R, Chmielarska K, Hennecke K, Pfeifer A, Fassler R. Cyld inhibits tumor cell proliferation by blocking BCl-3-dependent NF-kappaB signaling. Cell. 2006:125(4):665-77.

43. Park SG, Chung C, Kang H, Kim JY, Jung G. Up-regulation of Cyclin D1 by HBx Is Mediated by NF-B2/BCL3 Complex through kappaB Site of Cyclin D Promoter. J Biol Chem. 2006;281(42):31770-7.

44. Hsu SD, Tseng YT, Shrestha S, Lin YL, Khaleel A, Chou CH, et al. miRTarBase update 2014: an information resource for experimentally validated miRNA-target interactions. Nucleic Acids Res. 2014;42 (Database issue):D78-85.

45. Hediger MA, Clemencon B, Burrier RE, Bruford EA. The ABCs of membrane transporters in health and disease (SLC series): introduction. Mol Aspects Med. 2013;34(2-3):95-107.
46. Khananshvili D. The SLC8 gene family of sodium-calcium exchangers (NCX) structure, function, and regulation in health and disease. Mol Aspects Med. 2013;34(2-3):220-35.

47. Munoz JJ, Drigo SA, Barros-Filho MC, Marchi FA, Scapulatempo-Neto C, Pessoa GS, et al. Down-Regulation of SLC8A1 as a Putative Apoptosis Evasion Mechanism by Modulation of Calcium Levels in Penile Carcinoma. J Urol. 2014;194(1):245-51.

48. Januchowski R, Zawierucha P, Rucinski M, Andrzejewska M, Wojtowicz K, Nowicki M, et al. Drug transporter expression profiling in chemoresistant variants of the A2780 ovarian cancer cell line. Biomed Pharmacother. 2014;68(4):447-53.

49. Shiratsuchi T, Futamura M, Oda K, Nishimori H, Nakamura Y, Tokino T. Cloning and characterization of BAl-associated protein 1: a PDZ domaincontaining protein that interacts with BAl1. Biochem Biophys Res Commun. 1998;247(3):597-604.

50. Kotelevets L, van Hengel J, Bruyneel E, Mareel M, van Roy F, Chastre E. Implication of the MAGI-1b/PTEN signalosome in stabilization of adherens junctions and suppression of invasiveness. FASEB J. 2005;19(1):115-7.

51. Zhang G, Liu T, Wang Z. Downregulation of MAGI1 associates with poor prognosis of hepatocellular carcinoma. J Invest Surg. 2012;25(2):93-9.

52. Widenius M, Axmark D. Mysql Reference Manual. 1st ed. Paul DuBois. Sebastopol, CA USA: O'Reilly \& Associates Inc., 2002.

53. Griffiths-Jones S, Grocock RJ, Van Dongen S, Bateman A, Enright AJ. miRBase: microRNA sequences, targets and gene nomenclature. Nucleic Acids Res. 2006;34:D140-144.

54. Lewis BP, Burge CB, Bartel DP. Conserved seed pairing, often flanked by adenosines, indicates that thousands of human genes are microRNA targets. Cell. 2005;120(1):15-20.

\section{Submit your next manuscript to BioMed Central and we will help you at every step:}

- We accept pre-submission inquiries

- Our selector tool helps you to find the most relevant journal

- We provide round the clock customer support

- Convenient online submission

- Thorough peer review

- Inclusion in PubMed and all major indexing services

- Maximum visibility for your research 